\title{
DISPERSED IMAGES OF TOPOLOGICAL SPACES AND UNCOMPLEMENTED SUBSPACES OF $C(X)$
}

\author{
JOHN WARREN BAKER
}

\begin{abstract}
Let $I^{\prime}(\alpha)$ denote the set of ordinals not exceeding $\alpha$ with its interval topology. We show that, if $X$ is a 0 -dimensional Hausdorff space and $\alpha$ is a denumerable ordinal such that the $\alpha$ th derived set of $X$ contains $n$ points where $n<\omega$, there is a map of $X$ onto $\Gamma\left(\omega^{\alpha} \cdot n\right)$. Maps of completely regular spaces into the unit interval are considered and a noncompact analogue of a theorem of Pelczyński and Semadeni is obtained. Finally, these results are used to give a simple proof to the following theorem: If $X$ is completely regular and $X^{(\omega)} \neq \varnothing$, there is an uncomplemented subspace $H$ of $C(X)$ which is isometrically isomorphic to $C(Y)$ for some compact metric space $Y$.
\end{abstract}

Preliminaries. A topological space is called dispersed (scattered) if it does not contain a perfect subset. For any topological space $X$, we denote the set of accumulation points of $X$ by $X^{\prime}$. If $\lambda$ is an ordinal, the topological derivative of order $\lambda$, denoted $X^{(\lambda)}$, is defined by transfinite induction as follows:

$$
X^{(0)}=X, \quad X^{(\lambda+1)}=\left(X^{(\lambda)}\right)^{\prime}, \quad \text { and } \quad X^{(\lambda)}=\bigcap_{\beta<\lambda} X^{(\beta)}
$$

if $\lambda$ is a limit ordinal. The ordered pair $(\lambda, n)$ is called the characteristic (characteristic system) of $X$ if $n$ is a positive integer, $\lambda$ is an ordinal, and $X^{(\lambda)}$ contains exactly $n$ points. The symbol $\Gamma_{0}(\lambda)$ denotes the space of ordinals strictly less than $\xi$ with their interval topology. A topological space $X$ is called 0 -dimensional if its open-and-closed sets constitute a base for the topology. The first limit ordinal is denoted by (1) and the first uncountable ordinal by $\Omega$. If $\lambda$ is a nonlimit ordinal, then $\lambda-1$ denotes the predecessor of $\lambda$. We assume all maps are continuous and all spaces are Hausdorff.

The terminology in $\S 2$ follows that of Dunford and Schwartz, Linear operators. I [8]. In particular, if $X$ is a topological space, then $C(X)$ is the

Presented to the Society, January 26, 1973; received by the editors January 15, 1973. AMS (MOS) subject classifications (1970). Primary 54C05, 54F05, 46E15; Secondary 06A40, 06A45, 46B99, 46J10.

Key words and phrases. 0-dimensional spaces, dispersed spaces, scattered spaces, ordered topological spaces, Boolean spaces, Banach spaces of continuous functions, uncomplemented subspaces of $C(X)$.

(c) American Mathematical Society 1973 
Banach space of continuous bounded scalar-valued functions on $X$ with the supremum norm. If $\phi$ is a map of $X$ into a topological space $Y$, the induced operator $\phi^{\circ}$ from $C(Y)$ into $C(X)$ that takes $f \in C(Y)$ to $f \circ \phi \in C(X)$ is a multiplicative linear operator. For completely regular spaces, $\phi^{\circ}$ is an isometric isomorphism if and only if $\phi(X)$ is dense in $Y$ [10, p. 141]; moreover, if $\phi$ is a quotient map, then $\phi^{\circ}[C(Y)]$ is the set of all functions in $C(X)$ which are constant on each point inverse of $\phi[10, \mathrm{p} .146]$.

1. Dispersed images of topological spaces. Suppose $X$ is a compact dispersed space with characteristic $(\lambda, n)$. It is well known that the least ordinal $\alpha$ such that $\Gamma(\alpha)$ has characteristic $(\lambda, n)$ is $\alpha=\omega^{\lambda} \cdot n$. Example 1 in [5] shows that $X$ does not necessarily contain a subset homeomorphic to $\Gamma\left(\omega^{\lambda} \cdot n\right)$ even if $\lambda<\Omega$. However, if $\lambda<\Omega$, there is a map of $X$ onto $\Gamma\left(\omega^{\lambda} \cdot n\right)$; this fact is established by Theorem 1.1 and is valid for all 0 dimensional spaces $X$ with Card $X^{(\lambda)} \geqq n$.

THEOREM 1.1. If $X$ is 0-dimensional and $\lambda$ is a nonzero denumerable ordinal such that $X^{(\lambda)}$ contains at least $n$ points, there is a map of $X$ onto $\Gamma\left(\omega^{\lambda} \cdot n\right)$. Therefore, if $\lambda<\Omega, X$ is compact, and $X^{(\lambda)} \sim X^{(\lambda+1)}$ contains at least $n$ points, there is a map $\phi$ of $X$ onto $\Gamma\left(\omega^{\lambda} \cdot n\right)$.

Proof. Suppose $X$ is 0 -dimensional. Let $P(\alpha)$ denote the following statement: For every open-and-closed subset $Y$ of $X$ with $Y^{(a)} \neq \varnothing$, there is a map of $Y$ onto $\Gamma\left(\omega^{\alpha}\right)$. It is easy to prove $P(1)$. Suppose $\lambda$ is a denumerable ordinal with $\lambda \geqq 1$ and $P(\alpha)$ has been established for all $\alpha<\lambda$. Let $Y$ be an open-and-closed subset of $X$ with $y \in Y^{(\lambda)}$. If $\lambda$ is a limit ordinal, there is an increasing sequence $\left\{\alpha_{n}\right\}_{n=1}^{\infty}$ of ordinals less than $\lambda$ with $\lim _{n \rightarrow \infty} \alpha_{n}=\lambda$ (see [14, p. 382]). If $\lambda$ is a nonlimit ordinal, we let $\alpha_{n}=\lambda-1$ for each $n$. In either case, we define

$$
\pi_{0}=0, \quad \pi_{n}=\sum_{k=1}^{n} \omega^{\alpha_{k}}, \quad \text { and } \quad \pi=\sum_{k=1}^{\infty}(1)^{\alpha_{k}} .
$$

By induction, there is a decreasing sequence $\left\{U_{n}\right\}_{n=1}^{\infty}$ of open-and-closed subsets of $Y$ containing $y$ such that $K_{n}=U_{n} \sim U_{n+1}$ contains a point of $Y^{\left(\alpha_{n}\right)}$ for each $n$. We may suppose $U_{1}=Y$. Let $F=\bigcap_{n=1}^{\infty} U_{n}$. Since $K^{\left(\alpha_{n}\right)}$ is nonempty, by property $P\left(\alpha_{n}\right)$ there is a map $\phi_{n}$ of $K_{n}$ onto $\left(\pi_{n-1}, \pi_{n}\right]$. We define a function $\phi$ from $Y$ onto $\Gamma(\pi)$ by $\phi(x)=\phi_{n}(x)$ if $x \in K_{n}$ and $\phi(x)=$ $\pi$ if $x \in F$. It is easy to show that $\phi$ is a map of $Y$ onto $\Gamma(\pi)$.

It remains to be shown that $\pi=(1)^{\lambda}$. In case $\lambda$ is nonlimit, this is obvious. If $\lambda$ is a limit ordinal, then $(1)^{\lambda}=\lim _{\xi<\lambda}(1)^{\xi}$ so $\left.\pi_{n} \leqq(1)^{\alpha_{n}} \cdot n<(1)^{\alpha_{n}+1}<\omega\right)^{\lambda}$ and $\pi=\lim _{n \rightarrow \infty} \pi_{n} \leqq\left(\omega^{\lambda}\right.$. However, $\pi \geqq(1)^{\alpha_{n}}$ and hence $\pi \geqq \lim _{n \rightarrow \infty}(1)^{\alpha_{n}}=(1)^{\lambda}$. By transfinite induction, $P(\alpha)$ is valid for all $\alpha<\Omega$.

Suppose $\lambda<\Omega$ and $X^{(\lambda)}$ contains at least $n$ points, say $x_{1}, x_{2}, \cdots, x_{n}$. We partition $X$ into open-and-closed subsets $Y_{1}, Y_{2}, \cdots, Y_{n}$ so that 
$x_{k} \in Y_{k}$ for each $k$. Since $Y_{k}^{(\lambda)} \neq \varnothing$, we infer from the preceding property $P(\alpha)$ that there is a map $\psi_{1}$ of $Y_{1}$ onto $\left[0, \omega^{\lambda}\right]$ and a map $\psi_{k}$ of $Y_{k}$ onto the interval $\left(\omega^{\lambda} \cdot(k-1), \omega^{\lambda} \cdot k\right]$ for $1<k \leqq n$. Thus, $\psi=\bigcup_{k=1}^{n} \psi_{k}$ is a map of $X$ onto $\Gamma\left(\omega^{\lambda} \cdot n\right)$ and the first statement is established.

The second statement is an easy consequence of the first statement and the fact that each compact dispersed space is 0-dimensional [12].

Clearly, the "0-dimensional" assumption cannot be dropped from the hypothesis of Theorem 1.1, as this theorem is false for connected sets. The following example shows the "denumerable" requirement cannot be omitted from its hypothesis.

EXAMPLE 1.2. There is a dispersed compact (Hausdorff) space $X$ with characteristic $(\Omega, 1)$ which satisfies the following properties:

(a) There does not exist a map $\phi$ of a closed subspace $Y$ of $X$ into $\Gamma(\Omega)$ with $\phi(Y)^{(\Omega)} \neq \varnothing$.

(b) $X$ does not contain a subspace $Y$ homeomorphic to $\Gamma(\Omega)$.

Proof. Let $X^{*}$ be the free (disjoint) union of the spaces $\Gamma(\gamma)$ for $\gamma<\Omega$. Then $X^{*}$ is locally compact and has a one-point Hausdorff compactification $X$. If $c$ denotes the additional point in $X$, then a local base for the neighborhoods of $c$ consists of the sets $U_{F}=X \sim\left(\bigcup_{\alpha \in F} \Gamma(\alpha)\right)$ where $F$ is a finite subset of $\Omega$. Clearly $X^{(\Omega)}=\{c\}$, so $X$ has characteristic $(\Omega, 1)$.

Suppose there is a map $\phi$ of a closed subset $Y$ of $X$ into $\Gamma(\Omega)$ with $\phi(Y)^{(\Omega)} \neq \varnothing$. By Lemma 1 of [12], $\{\Omega\}=\phi(Y)^{(\Omega)} \subset \phi\left(Y^{(\Omega)}\right) \subset\{\phi(c)\}$ so $\phi(c)=\Omega$. (Note in [12] there is a misprint and the subset notation should be reversed.) Since $Y \sim \phi^{-1}(\Omega)=\phi^{-1}(\phi(Y) \sim\{\Omega\})$ is uncountable, there is a sequence $\left\{\alpha_{n}\right\}_{n=1}^{\infty}$ of distinct ordinals with $y_{n}$ in $\left(Y \sim \phi^{-1}\{\Omega\}\right) \cap \Gamma\left(\alpha_{n}\right)$. But, $\lim y_{n}=c$, so $\lim _{n \rightarrow \infty} \phi\left(y_{n}\right)=\Omega$, which is impossible. The proof of (b) is similar.

In [12], Pełczyński and Semadeni show that if $X$ is a nondispersed, compact space, there is a map $\phi$ of $X$ onto $[0,1]$. The following theorem is a noncompact analogue of this theorem which is also valid for dispersed spaces.

THEOREM 1.3. Let $X$ be completely regular and $\lambda$ be a nonzero denumerable ordinal. If $X^{(\lambda)}$ contains $n$ points, there is a map $\phi$ of $X$ into $[0,1]$ such that $\phi(X)^{(\lambda)}$ contains at least $n$ points. If $\lambda$ is a limit ordinal and $X^{(\gamma)} \neq \varnothing$ for $\gamma<\lambda$, there is a map $\phi$ of $X$ into $[0,1]$ with $\phi(X)^{(\lambda)} \neq \varnothing$.

Proof. Suppose $\beta(X)$, the Stone-Cech compactification of $X$, is not dispersed. By Theorem 1 in [12], there is a map $\psi$ of $\beta X$ onto [0,1]. The restriction $\phi$ of $\psi$ to $X$ is a map of $X$ onto a dense subset of $[0,1]$; hence $\phi(X)^{(\lambda)}=[0,1]$ and both conclusions of the theorem are obvious.

Next, suppose $\beta X$ is dispersed. Then $\beta X$ is 0 -dimensional [12]. In either case, $\beta X^{(\lambda)} \neq \varnothing$. In case 1 , let $m=n$ and in case 2 , let $m=1$. Since $[0,1]$ 
contains a subset $K$ homeomorphic to $\Gamma\left((1)^{\lambda} m\right)$, it follows by Theorem 1.1 that there is a map $\psi$ of $\beta X$ onto $K$. The restriction $\phi$ of $\psi$ to $X$ is a map of $X$ onto a dense subset of $K$; hence, $\phi(X)^{(\lambda)}=K^{(\lambda)}$. Since $K^{(\lambda)}$ contains $m$ points, this establishes both conclusions.

If $X$ is compact, the map $\phi$ in Theorems 1.1 and 1.3 is closed. The following example shows that, in general, $\phi$ may not even be a quotient map. According to Corollary 3 in [15] each open cover of the space $X_{\gamma}$ in Example 1.4 has a disjoint open refinement; hence, all dimensions (ind, Ind and dim) are zero. The construction is similar to that of the ordinal numbers, except $\Omega$ is used as the basic building block instead of 1 .

EXAMPLE 1.4. For each ordinal $\gamma$, there is a dispersed, Lindelöf, normal (hence, paracompact) space $X_{\gamma}$ with characteristic $(\gamma, 1)$ such that each map of $X_{\gamma}$ into a first-countable space has open point inverses and countable range. In particular, each quotient map of $X$ onto a compact, first-countable space has finite range.

Proof. First, we let $X_{0}=\{1\}$. Suppose $\gamma \geqq 1$ is an ordinal and $X_{\lambda}$ has been constructed for $\lambda<\gamma$. Let $I=\Gamma_{0}(\Omega) \times \Gamma_{0}(\gamma)$. Let $S=\{Y(\alpha, \beta) \mid(\alpha, \beta) \in I\}$ be a pairwise disjoint family of sets with each $Y(\alpha, \beta)$ homeomorphic to $X_{\beta}$ for $\alpha<\Omega$. Let $p$ be a point not in any $Y(\alpha, \beta)$ and let $X_{\gamma}$ consist of the point $p$ and the free union of the sets in $S$ where a neighborhood base for $p$ is given by the sets $U_{F}=X_{\gamma} \sim(\bigcup\{Y(\alpha, \beta) \mid(\alpha, \beta) \in F\})$ and $F$ is a countable subset of $I$.

It is obvious that $X_{\gamma}$ is Hausdorff with characteristic $(\gamma, 1)$. Let $\left\{G_{\mu}\right\}_{\mu \in M}$ be an open cover of $X_{\gamma}$. Select $t \in M$ such that $p \in G_{t}$. Then, $U_{F} \subset G_{t}$ for some countable subset $F$ of $I$. Since $X_{\gamma} \sim G_{t} \subseteq \bigcup\{Y(\alpha, \beta) \mid(\alpha, \beta) \in F\}$ and each $Y(\alpha, \beta)$ is Lindelöf by hypothesis, there is a countable subcover of $\left\{G_{\mu}\right\}_{\mu \in M}$ which covers $X_{\gamma} \sim G_{t}$. Hence, $X_{\gamma}$ is Lindelöf.

Let $A$ and $B$ be closed disjoint subsets of $X_{\gamma}$. If $p \in A$, there exists a neighborhood $U_{F}$ of $p$ with $U_{F} \cap B=\varnothing$. Thus, $B \subseteq \bigcup\{Y(\alpha, \beta) \mid(\alpha, \beta) \in F\}$. As $A \cap Y(\alpha, \beta)$ and $B \cap Y(\alpha, \beta)$ are closed, disjoint subsets of the normal space $Y(\alpha, \beta)$, there exist open, disjoint subsets $V(\alpha, \beta)$ and $W(\alpha, \beta)$ of $Y(\alpha, \beta)$ such that $A \cap Y(\alpha, \beta) \subset V(\alpha, \beta)$ and $B \cap Y(\alpha, \beta) \subset W(\alpha, \beta)$, $V=(\bigcup\{V(\alpha, \beta) \mid(\alpha, \beta) \in F\}) \cup U_{F}, W=\bigcup\{W(\alpha, \beta) \mid(\alpha, \beta) \in F\}$ are disjoint open sets containing $A$ and $B$, respectively. If $p \notin A \cup B$, there exists a neighborhood $U_{k}$ of $p$ with $(A \cup B) \cap U_{K}=\varnothing$. In this case $V=$ $\bigcup\{V(\alpha, \beta) \mid(\alpha, \beta) \in K\}$ and $W=\bigcup\{W(\alpha, \beta) \mid(\alpha, \beta) \in K\}$ are disjoint open sets containing $A$ and $B$, respectively. Thus, $X_{\gamma}$ is normal.

Let $\phi$ be a map of $X_{\gamma}$ into a first-countable space $Y$. Let $g=\phi(p)$ and let $\left\{G_{n}\right\}_{n=1}^{\infty}$ be an open neighborhood base for $g$. Since $T_{n}=\left\{x \in X_{\gamma} \mid f(x) \notin G_{n}\right\}$ is closed and does not contain $p$, there is a countable subset $F_{n}$ of $I$ with $T_{n} \cap U_{F_{n}}=\varnothing$. If $F=\bigcup_{n=1}^{\infty} F_{n}$ then $F$ is a countable subset of $I, U_{F}$ is a neighborhood of $p$, and $\phi^{-1}(y) \subset \bigcup\{Y(\alpha, \beta) \mid(\alpha, \beta) \in F\}$ for each 
$y \in(Y \sim\{g\})$. By hypothesis, $\phi^{-1}(g)=\left(\bigcup\left\{\phi^{-1}(g) \cap Y(\alpha, \beta) \mid(\alpha, \beta) \in F\right\}\right) \cup U_{F}$ is open and $\phi^{-1}(y)=\bigcup\left\{\phi^{-1}(y) \cap Y(\alpha, \beta) \mid(\alpha, \beta) \in F\right\}$ is open for $y \neq g$. Consequently, $\phi^{-1}(y)$ is open for each $y \in Y$. The proof that the range of $\phi$ is countable is similar.

2. Uncomplemented subspaces of $C(X)$. In [1], D. Amir proved that $C[0,1]$ contains an uncomplemented subspace isometrically isomorphic to $C[0,1]$. R. Arens established in [2] that there is a countable, compact, metric space $X$ and a closed subspace $S$ of $C(X)$ isometrically isomorphic to $C(Y)$ for a quotient space $Y$ of $X$ such that $S$ is not complemented in $C(X)$. It can easily be seen from the construction of $X$ that $X^{(\omega)}$ contains exactly one point. By a theorem due to Mazurkiewicz and Sierpiński (see $\left[11\right.$, p. 103] or [4]), $X$ is homeomorphic to $\Gamma\left(\omega^{\omega}\right)$. By the same argument, $Y$ is also homeomorphic to $\Gamma\left(\omega^{\omega}\right)$.

The next theorem was established for normal spaces in [3]. The author wishes to thank Professor Lindenstrauss for suggesting this technique of proof.

THEOREM 2.1. Suppose $X$ is completely regular and $X^{(n)} \neq \varnothing$ for $n<\omega$. There exist a compact metric space $Y$ and a map $\phi$ of $X$ onto $Y$ such that $\phi^{\circ}$ is a multiplicative isometric isomorphism from $C(Y)$ onto an uncomplemented selfadjoint subalgebra of $C(X)$.

Proof. Clearly $\beta X^{(\omega)} \neq \varnothing$. First suppose $\beta X$ is dispersed. By Theorem 1.1 , there is a map $\psi$ of $\beta X$ onto $\Gamma\left(\omega^{\omega}\right)$. Let $\phi=\psi \mid X$. Since $\phi(X)$ is dense in $\Gamma\left(\omega^{\omega}\right), \phi^{\circ}$ is a multiplicative isometric isomorphism from $C\left(\Gamma\left(\omega^{\omega}\right)\right)$ into $C(X)$ [10, Theorem 10.3]. By Theorem 4.1 in [6], there is a map $\psi$ of $\Gamma\left(\omega^{\omega}\right)$ onto itself such that $S=\psi\left[C\left(\Gamma\left(\omega^{\omega}\right)\right)\right]$ is uncomplemented in $C\left(\Gamma\left(\omega^{\omega}\right)\right)$. Then $A=\phi^{\circ}(S)$ is uncomplemented in $C(X)$ since a projection $P$ of $C(X)$ onto $A$ would induce a projection $\left(\phi^{\circ}\right)^{-1} P \phi^{\circ}$ of $C\left(\Gamma\left(\omega^{\omega}\right)\right)$ onto $S$.

If $\beta X$ is not dispersed, there is a map $\psi$ of $\beta X$ onto $[0,1]$. As before, if $\phi=\psi \mid X$, then $\phi^{\circ}$ is an isometry from $C([0,1])$ into $C(X)$. By Corollary 5.8 of [7], there is a map $\psi$ of $[0,1]$ onto itself such that $H=\psi^{\circ}[C([0,1])]$ is uncomplemented in $C([0,1])$. It follows that $A=\phi^{\circ}(H)$ is an uncomplemented subalgebra of $C(X)$.

If, in the preceding proof, we drop our dependence upon [6] and [7] by letting $S$ be the uncomplemented subspace of $C\left(\Gamma\left(\omega^{\omega}\right)\right)$ constructed by Arens and by letting $H$ be the uncomplemented subspace of $C[0,1]$ constructed by Amir, we obtain an easy proof for the following theorem: If $X$ is completely regular and $X^{(n)} \neq \varnothing$ for $n<\omega$, then there exists an uncomplemented subspace of $C(X)$ which is isometrically isomorphic to $C(Y)$ for some compact metric space $Y$. 


\section{BIBLIOGRAPHY}

1. D. Amir, Continuous function spaces with the bounded extension property, Bull. Res. Council Israel Sect. F 10F (1962), 133-138. MR 26 \#592.

2. R. Arens, Projections on continuous function spaces, Duke Math. J. 32 (1965), 469478. MR 31 \#6108.

3. J. W. Baker, Some uncomplemented subspaces of $C(X)$ of the type $C(Y)$, Studia Math. 36 (1970), 85-103. MR 43 \#1113.

4. - Compact spaces homeomorphic to a ray of ordinals, Fund. Math. 76 (1972), $19-27$.

5. - Ordinal subspaces of topological spaces, General Topology and Appl. 3 (1973), 85-91.

6. - Uncomplemented $C(X)$-subalgebras of $C(X)$, Trans. Amer. Math. Soc. (to appear).

7. S. Ditor, Averaging operators in $C(S)$ and lower semicontinuous sections of continuous maps, Trans. Amer. Math. Soc. 175 (1973), 195-208.

8. N. Dunford and J. T. Schwartz, Linear operators. I: General theory, Pure and Appl. Math., vol. 7, Interscience, New York, 1958. MR 22 \#8302.

9. R. Engelking, Outline of general topology, PWN, Warsaw, 1965; North-Holland, Amsterdam; Interscience, New York, 1968. MR 36 \#4508; MR 37 \#5836.

10. L. Gillman and M. Jerison, Rings of continuous functions, University Series in Higher Math., Van Nostrand, Princeton, N.J., 1960. MR 22 \#6994.

11. K. Kuratowski, Topologie, Vol. II, 3rd ed., Monographie Mat., Tom 21, PWN, Warsaw, 1961; English transl., Academic Press, New York; PWN, Warsaw, 1968. MR 24 \#A2958; 41 \#4467.

12. A. Pelczyński and Z. Semadeni, Spaces of continuous functions. III, Spaces $C(\Omega)$ for $\Omega$ without perfect subsets, Studia Math. 18 (1959), 211-222. MR 21 \#6528.

13. Z. Semadeni, Sur les ensembles clairsemés, Rozprawy Mat. 19 (1959), 1-39. MR 21 \#6571.

14. W. Sierpiński, Cardinal and ordinal numbers, 2 nd rev. ed., Monografie Mat., Vol. 34, PWN, Warsaw, 1965. MR 33 \#2549.

15. R. Telgársky, Total paracompactness and paracompact dispersed spaces, Bull. Acad. Polon. Sci. Sér. Sci. Math. Astronom. Phys. 16 (1968), 567-572. MR 38 \#3826.

Department of Mathematics, Florida State University, Tallahassee, Florida 32306 